\title{
DNS of compressible turbulent boundary layer over a blunt wedge
}

\author{
LI Xinliang, FU Dexun \& MA Yanwan \\ LNM, Institute of Mechanics, Chinese Academy of Sciences, Beijing 100080, China \\ Correspondence should be addressed to Li Xinliang (email: lixl@Inm.imech.ac.cn) \\ Received May 10, 2004
}

\begin{abstract}
Direct numerical simulation of spatially evolving compressible boundary layer over a blunt wedge is performed in this paper. The free-stream Mach number is 6 and the disturbance source produced by wall blowing and suction is located downstream of the sound-speed point. Statistics are studied and compared with the results in incompressible flat-plate boundary layer. The mean pressure gradient effects on the vortex structure are studied.
\end{abstract}

Keywords: boundary layer, transition, direct numerical simulation, compressible turbulence.

DOI: 10.1360/03yw0300

Wall turbulence is more complicated than free turbulence, and the direct numerical simulation (DNS) of wall turbulence is more difficult. In recent years, most of DNS cases for wall turbulence are simplified by using temporal mode, where streamwise periodic boundary condition is imposed. In temporal mode, spatial transition will be considered as an analogue of time-evolving transition. For the channel turbulence, an equivalent body force can substitute for the mean gradient of pressure, and then the streamwise periodic boundary condition can be used. Because of evolving of the boundary layer, there is no streamwise periodic property, but the evolving of the boundary layer can be replaced by an equivalent body force and then the periodic condition can be used. The temporal mode can only be used in relatively simple flow, such as channel flow or flat plate boundary layer, but cannot be used in the flow with complex shape, such as flow over a blunt body. Compared with the temporal mode, there is no artificial assumption in spatial mode, and the result seems more reliable. Because of the high cost of computation for spatial mode, only a few papers on DNS of spatially evolving boundary layer have been published.

Compared with incompressible turbulence, DNS for compressible turbulence started much later. Guarini et al. ${ }^{[1]}$ performed DNS of a flat-plate boundary layer with free stream Mach number 2.5, and Maeder et al. ${ }^{[2]}$ performed the same DNS with free stream Mach number 3, 4.5 and 6. Compressibility effects are studied in those two pa- 
pers. According to Maeder et al. ${ }^{[2]}$, the maximum turbulent Mach number for the boundary layer with $M_{\infty}=6$ is 0.45 , and Morkovin's theory is not valid. Both Guarini and Maeder used temporal mode in their numerical work. Rai et al. ${ }^{[3]}$ performed DNS for spatially evolving flat-plate boundary at free stream Mach number 2.5, and studied the statistics of turbulence.

Most of the DNS work conducted is of flat-plate boundary layer. But the boundary layer of a blunt body is more interesting in aeronautics and astronautics. The prediction of laminar-turbulent transition in hypersonic boundary layers is a critical part of the aerodynamic design and control of hypersonic vehicles ${ }^{[4]}$. Zhong et al. ${ }^{[4]}$ performed numerical simulation of leading-edge receptivity to freestream disturbance waves for hypersonic flow over a body with parabolic shape, but the simulation was limited to two-dimensional. Laurien ${ }^{[5]}$ performed analysis and DNS of boundary layer of a re-entry capsule. But the DNS in Laurien's work is on temporal evolving flat-plate boundary layer. There is still no DNS work on the turbulent boundary layer of a blunt body.

Direct numerical simulation of spatially evolving compressible boundary layer over a blunt wedge with the free stream Mach number 6 is performed in this paper. The disturbance source produced by blowing and suction is located at the wall downstream of the sound-speed point. Statistics are studied and compared with the result for incompressible flat-plate boundary layer. The mean pressure gradient effects on the vortex structure are studied. Because of existence of bow shock, the turbulent Mach number in turbulent boundary layer is not high, and the compressible effects are not significant. It is also found that near-wall disturbance is strongly suppressed in the near nose region of the blunt wedge, but the suppression of far-from-wall disturbance is not significant.

\section{Numerical simulation}

A schematic diagram of supersonic flow over a blunt wedge is shown in fig. 1. The half wedge angle is $\theta$, and free stream Mach number is defined as $M_{\infty}=u_{\infty} / c_{\infty}$, and the Reynolds number is $R e=\rho_{\infty} u_{\infty} R / \mu_{\infty}$. We suppose that the temperature on the wall $T_{w}$ is constant. The flow parameters are given in table 1 .

Table 1 Flow parameters

\begin{tabular}{cccc}
\hline$M_{\mathbf{-}}$ & $\theta$ & $R e$ & $T_{w}$ \\
\hline 6.0 & $6^{\circ}$ & $2.0 \times 10^{6}$ & 5.0 \\
\hline
\end{tabular}

At the first step, numerical simulation of two-dimensional steady laminar flow is performed, and the numerical results are used in the initial condition and the inlet and outer boundary condition of three-dimensional computation.

\subsection{Numerical simulation of two-dimensional flow}

At the first step, two-dimensional steady flow is numerically simulated. The governing equations are the two dimensional compressible Navier-Stokes equations, and no Copyright by Science in China Press 2005 


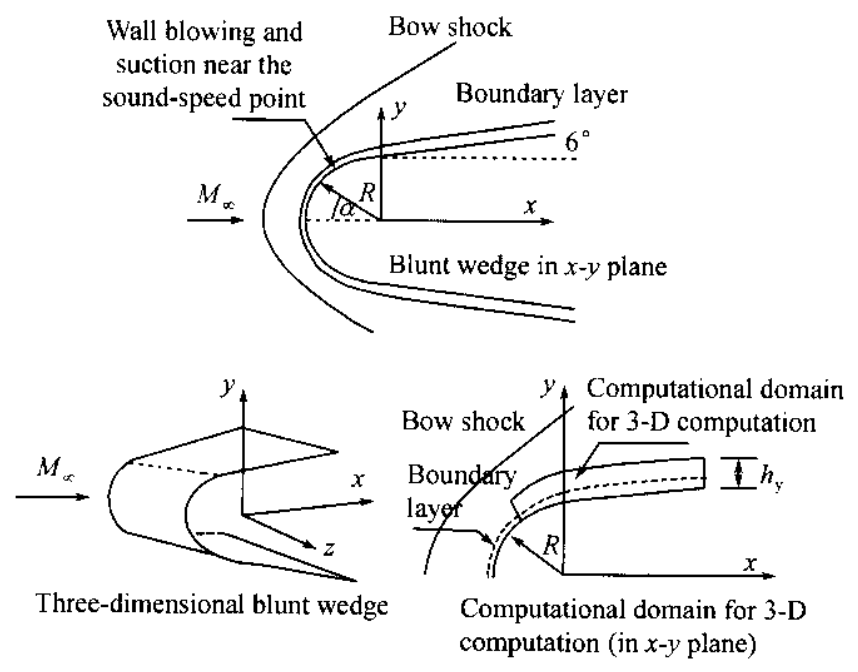

Fig. 1. Supersonic flow over a blunt wedge.

slip boundary conditions are used at the wall. The uniform free flow condition is used at the outflow, the symmetry condition is used in the symmetry plane, and the no-reflex outflow condition is used at the outflow boundary.

The viscous terms of the Navier-Stokes equations are discretized with 6th order central difference approximation. The flux vector splitting is adopted for the convection terms. The 5th order WENO schemes ${ }^{[6]}$ are used to approximate convection terms. The grid number is $1080 \times 380$.

Contours of pressure in the two-dimensional steady flow are shown in fig. 2, from which a bow shock over the blunt wedge can be seen clearly. Contours of Mach number are shown in fig. 3. Form this figure we can see that the maximum Mach number inside the bow shock is 2.5, which is much less than the free-stream Mach number. Because of existence of bow shock, the turbulent Mach number in turbulent boundary layer is not so high, and the compressible effects are not significant.

Fig. 4 shows the pressure distribution along the wall, where the abscissa is $\alpha$ (the definition of $\alpha$ is shown in fig. 1), and the ordinate is $p / p_{\omega}$. In fig. 4 , the solid line denotes computational results and symbols denote the results in ref. [7] for the case of $M_{\infty}$ = 6 flow over a column. When $M_{\infty}=6$, the sound-speed-point is located at the head of the blunt wedge, so the wall pressure of the head is very close to the wall pressure of the column. From this figure we can see the two results agree with each other very well.

There is curvature discontinuity at the edge between the head and the body of the blunt wedge, and this curvature discontinuity has some effects on the flow. Fig. 5 shows the wall pressure near the curvature discontinuity point $\left(\alpha=84^{\circ}, x=-0.105\right)$. There is very strong favorable pressure gradient at the nose of the blunt wedge, and there is 


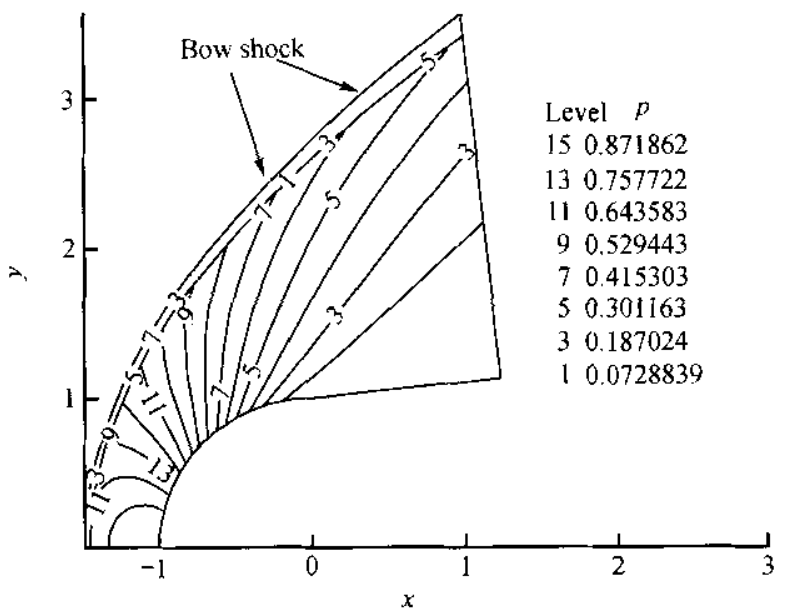

Fig. 2. Contours of pressure in two-dimensional steady flow.

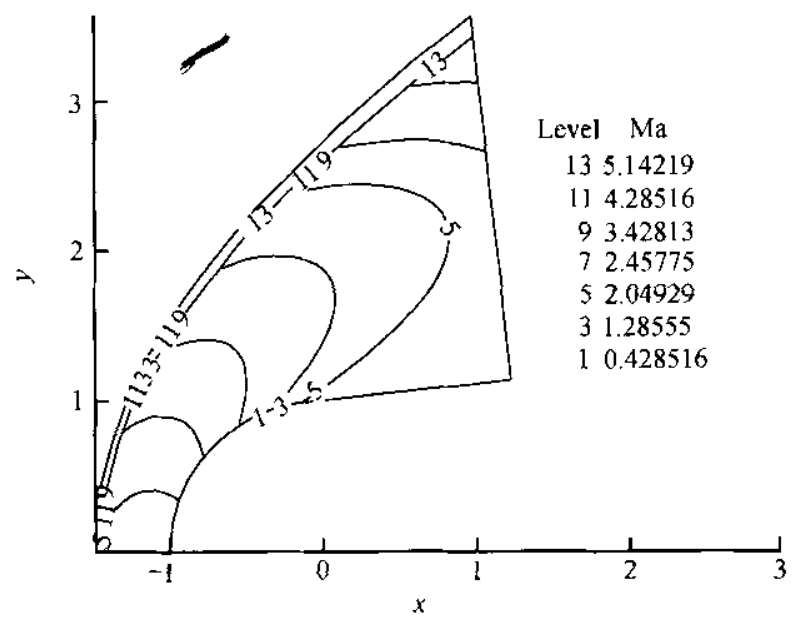

Fig. 3. Contour of Mach number in two-dimensional steady flow. Ma, Mach number.

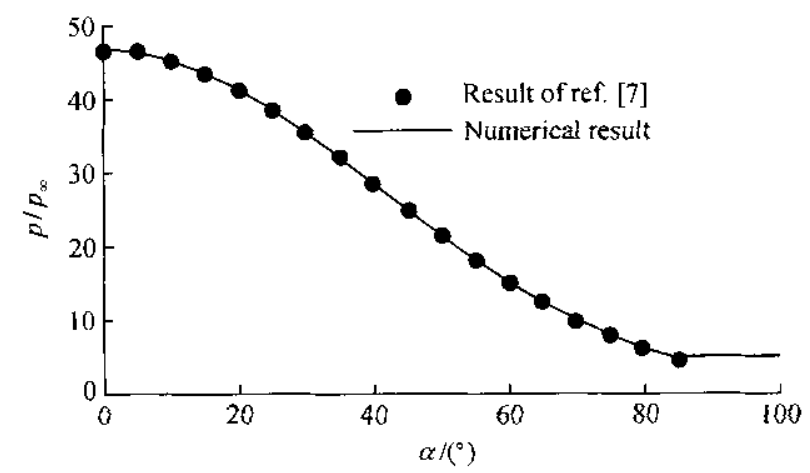

Fig. 4. Pressure distribution along the wall.

Copyright by Science in China Press 2005 


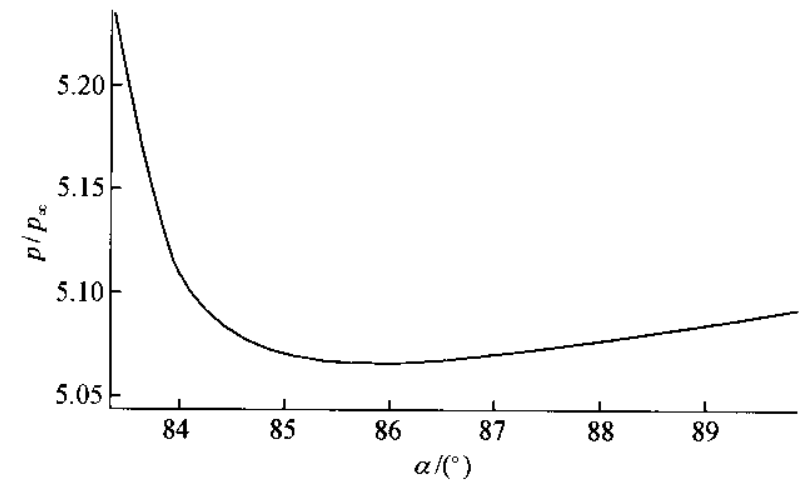

Fig. 5. Pressure distribution along the wall near the curvature discontinuity.

adverse pressure gradient at the body of the blunt wedge.

\subsection{Three-dimensional simulation}

The 3-D computational domain (see fig. 1) is shown in table 2. The governing equations are Jocabian-transformed Navier-Stokes equations ${ }^{[9]}$, and Sutherland equation is used to calculate the viscous coefficient. The viscous terms of the Navier-Stokes equations are discretized with a 6th order central difference. The flux vector splitting is used for the inviscous terms, and 7th order accurate upwind-biasing difference approximations are used to discretize the inviscous terms. Three-order Range-Kutta method is used for time advancing.

Table 2 Computational domain of three-dimensional numerical simulation

\begin{tabular}{ccc}
\hline Streamwise & Vertical & Spanwise \\
\hline$-0.628 \leqslant x \leqslant 1.224$ & $h_{y}=0.134$ & $h_{z}=0.1$ \\
\hline
\end{tabular}

Non-slip and isothermal boundary conditions are used at the wall. The density, velocity and temperature are invariable at the inlet boundary and outer boundary, and the values are obtained from two-dimensional simulation. Supersonic non-reflecting outflow boundary condition is used at the outlet section.

The blow and suction disturbance is imposed at the wall of $-0.5735 \leqslant x \leqslant-0.5062$, and the form of the disturbance is shown as follows:

$$
\begin{aligned}
& v_{b s}=A u_{\infty} f(x) g(z) h(t), \\
& f(x)=4 \sin \theta(1-\cos \theta) /(27)^{1 / 2}, \quad \theta=2 \pi\left(x-x_{a}\right) /\left(x_{b}-x_{a}\right), \\
& g(z)=\sum_{l=0}^{l_{\max }} Z_{l} \sin \left(2 \pi l\left(z l z_{\max }+\phi_{l}\right)\right), \quad \sum_{l=0}^{l_{\max }} Z_{l}=1, \quad Z_{l}=1.25 Z_{l+1},
\end{aligned}
$$




$$
h(t)=\sum_{m=0}^{m_{\max }} T_{l} \sin \left(2 \pi m\left(\beta t+\phi_{m}\right)\right), \quad \sum_{m=0}^{m_{\max }} T_{m}=1, \quad T_{m}=1.25 T_{m+1},
$$

where $l_{\max }=10, m_{\max }=5, x_{a}, x_{b}$ are the beginning and the end of disturbance range on the $x$ axis, respectively. $z_{\max }$ is the length of spanwise computational domain. $\phi_{l}, \phi_{m}$ are random numbers between 0 and $1 . A=0.04$ is amputate and $\beta$ is base frequency.

Computational parameters are shown in table 3. Programs are coded by using MPI Fortran, and the DNS results are computed with computer LSSC2 of LSEC (State Key Laboratory of Scientific and Engineering Computing). Averaged performance is 8.3 seconds/time-step (40 CPUs in 20 nodes).

Table 3 Computational parameters

\begin{tabular}{ccccc}
\hline$R e$ & $M_{-}$ & $T_{w}$ & $\beta$ & Grid number \\
\hline $2.0 \times 10^{6}$ & 6.0 & 5.0 & 2.5 & $800 \times 160 \times 80$ \\
\hline
\end{tabular}

\section{Result analysis}

The two-dimensional steady flow is used as the initial condition of three-dimensional computation, and three-dimensional computation is performed until the turbulence reached equilibrium.

Averaged velocity and temperature of three sections near the inlet are shown in fig. 6 and fig. 7, respectively. The three sections are all normal to the wall and located at $x=$ $0.628,-0.604$ and -0.580 (the streamwise index of computational grid in $i=10,20$ and 30 , respectively). In the two figures and the remainder of the paper, $y$ denotes the vertical distance to the wall. It can be seen that the thickness of velocity boundary layer in the inlet is 0.004 and thickness of the temperature boundary layer is 0.0005 .

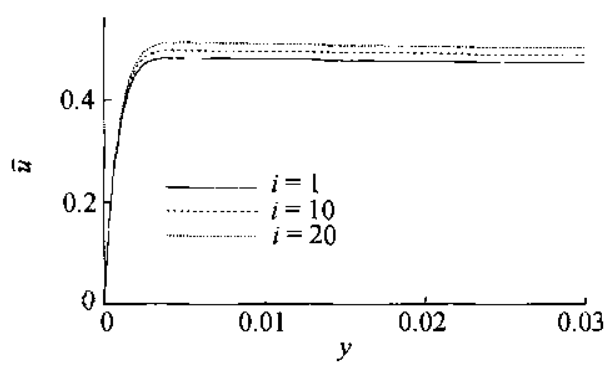

Fig. 6. Distribution of averaged velocity at the three sections near the inlet.

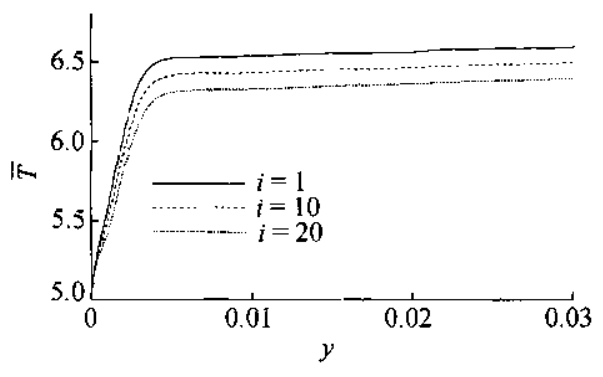

Fig. 7. Distribution of averaged temperature at the three sections near the inlet.

RMS of velocity fluctuation $V_{r m s}^{\prime}=\sqrt{u_{r m s}^{\prime 2}+v_{r m s}^{\prime 2}+w_{r m s}^{\prime 2}}$ at five sections normal to the wall located at $x=-0.370,-0.094,0.172,0.419$ and 0.852 are given in fig. 8 . We can see the disturbance outside the boundary layer is very low. 


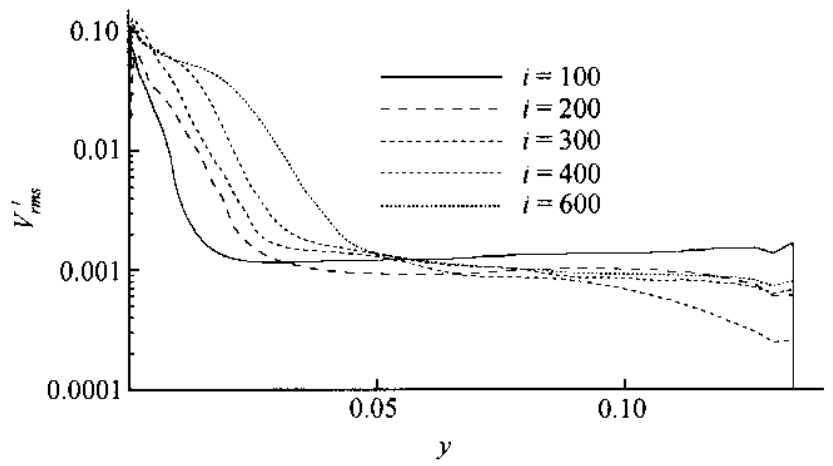

Fig. 8. Variation of RMS of velocity fluctuation at five sections normal to the wall.

Fig. 9 shows the skin friction in the streamwise direction. It can be seen that skin friction increases fast in the transition region. The skin friction is defined as

$$
C_{f}=\tau_{w} / \frac{1}{2} \rho_{\infty} u_{\infty}^{2}, \tau_{w}=\left.\mu_{w} \frac{\partial \bar{u}}{\partial y}\right|_{w} .
$$

Fig. 10 shows the spanwise energy spectra $\hat{E}(k, x)$ as a function of $x$ at $y_{0}=$ $0.0002\left(y_{0}^{+}=4.2\right)$, and $k=4,8,16$ and 24. Here $\hat{E}(k, x)=\frac{1}{h_{z}} \int E\left(x, y_{0}, z\right) e^{-i k z} d z, E=$ $\frac{1}{2}\left(u^{2}+v^{2}+w^{2}\right)$.

Fig. 9 and fig. 10 show that transition occurs soon after the disturbance zone, and then transition is depressed at the head of the blunt-wedge, and finished at the body of the blunt-wedge.

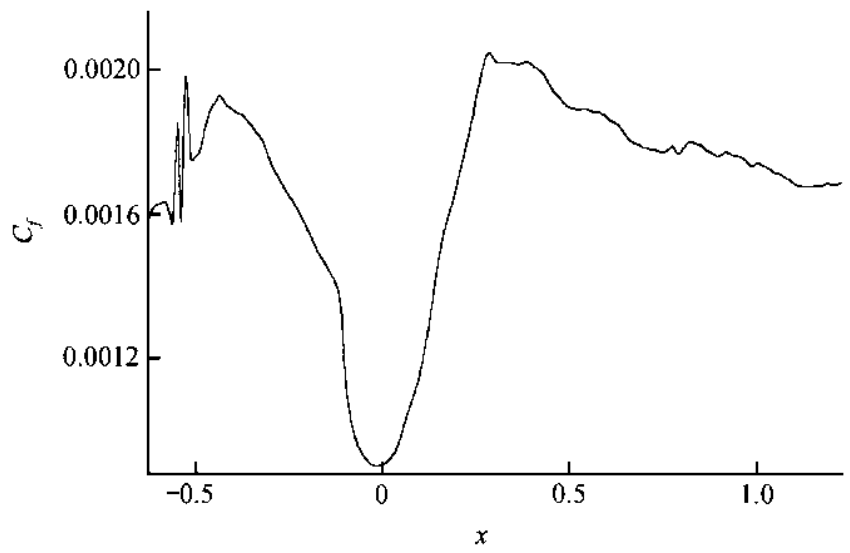

Fig. 9. Skin friction in the streamwise direction. 


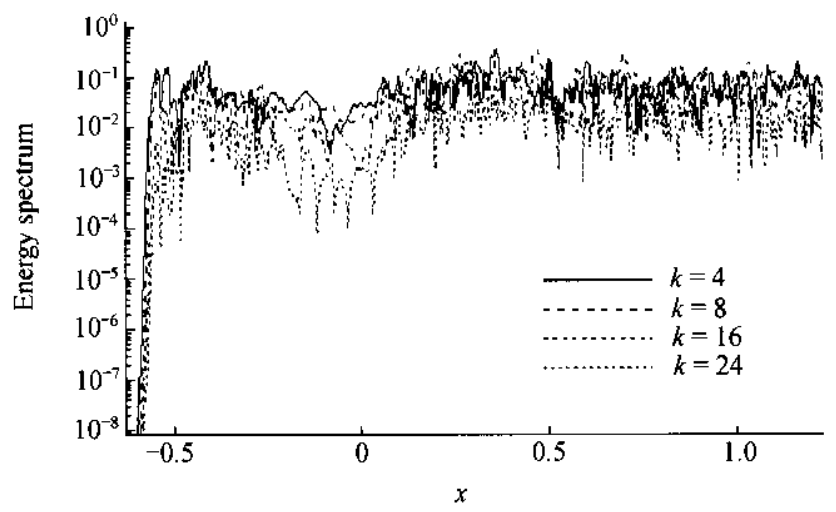

Fig. 10. Spanwise energy spectra vary as a function of $x$.

Fig. 11 shows the mean Van Driest velocity profile ${ }^{[3]}$ normalized by wall-shear velocity at $x=1.0$. The computed results obey the wall law and log law. The log law is expressed as $u^{+}=1 / \kappa \ln y^{+}+C$, where $\kappa=0.4, C=7.8$. Because of existence of stream pressure gradient, the coefficient $C$ is different from that in the incompressible flat-plate boundary layer where $C=5.5$.

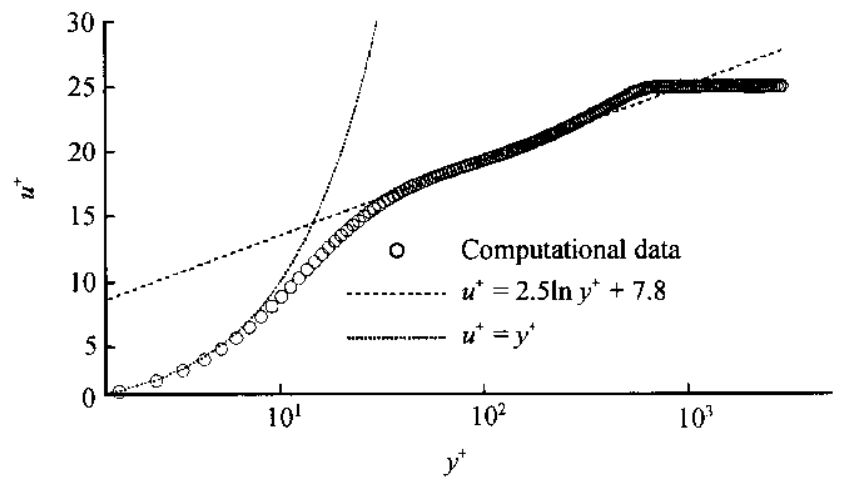

Fig. 11. Mean Van Driest velocity.

Fig. 12 shows the RMS of velocity fluctuation $u_{r m s}^{\prime}, v_{r m s}^{\prime}, w_{r m s}^{\prime}$ at $x=1.0$ as a function of the vertical distance to the wall. We can see that the peak RMS is located at $y^{+}=20$. In the near-wall zone $u_{r m s}^{\prime}>w_{r m s}^{\prime}>v_{r m s}^{\prime}$, which means that the turbulence is strong anisotropic near the wall. In the far-from-wall zone, difference between $u_{r m s}^{\prime}$, $v_{r m s}^{\prime}$ and $w_{r m s}^{\prime}$ is not large, which means that the turbulence in the far-from-wall zone is nearly isotropic.

Fig. 13 shows the turbulent Mach number defined by $M_{t}=\frac{\sqrt{\overline{u^{\prime 2}+v^{\prime 2}+w^{\prime 2}}}}{\bar{c}}$ at the 


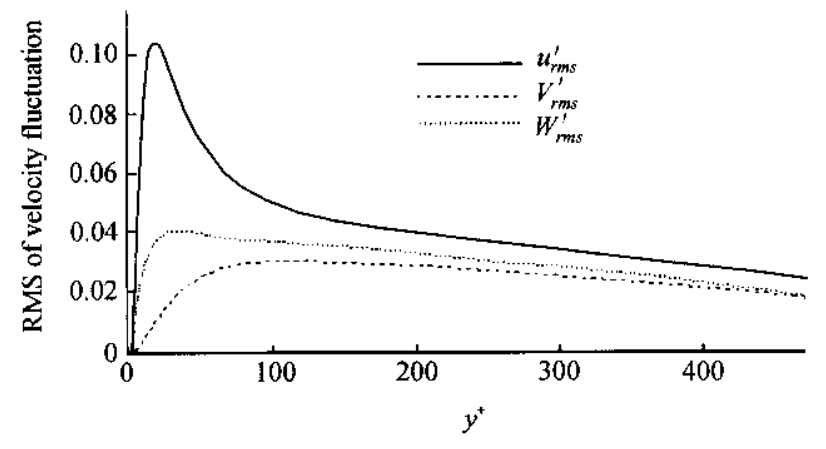

Fig. 12. RMS of velocity fluctuation at $x=1.0$.

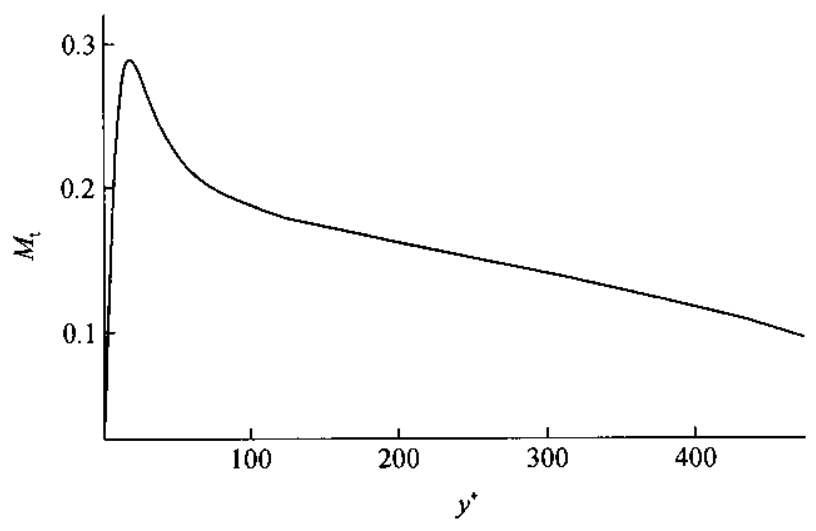

Fig. 13. Distribution of turbulent Mach number at $x=1.0$.

location $x=1.0$ as a function of vertical distance to the wall $y$. It can be seen that the peak of the turbulent Mach number equal to 0.29 is located at $y^{+}=20$, which is much less than 0.45 in the flat-plate boundary layer for the same free stream Mach number $M_{\infty}$ $=6$. This is because of existence of strong bow shock out of the boundary layer. Fig. 14 and fig. 15 show the normalized turbulence intensities and normalized Reynolds shear stress at $x=1.0$, where the symbols denote the experiment data for incompressible flat-plate boundary. Because the turbulent Mach number is not so high, the difference between those two data is not significant.

Fig. 16 shows the isosurface of streamwise vorticity $\omega_{s}= \pm 80$ at $0.03 \leqslant x \leqslant 0.23$, from which coherent structure can be seen in the transition region.

Fig. 17 shows turbulent intensity $V_{r m s}^{\prime}$ at $y=0.00020,0.00043,0.00102$ and $0.00180\left(y^{+}=4.2,9.1,21.2\right.$ and 37.6) as a function of streamwise coordinate $x$. We can see that the turbulent intensity increases fast after the disturbance zone, and it means that transition occurs, and then the turbulent intensity is depressed at the head of the 


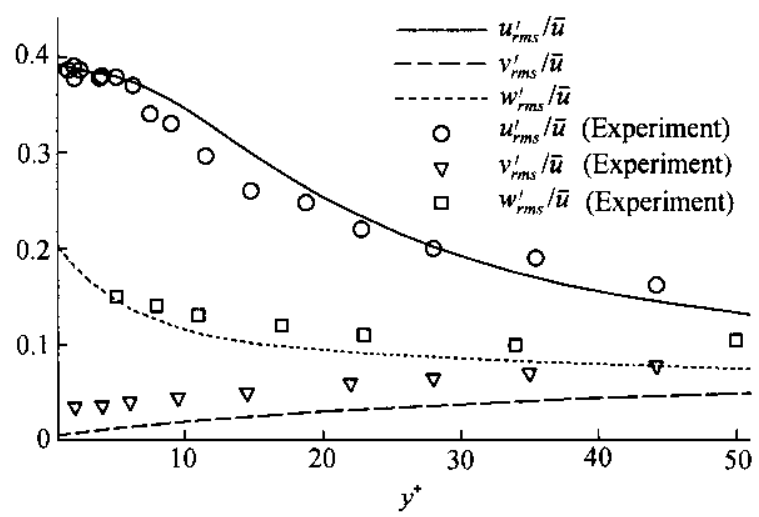

Fig. 14. Normalized RMS velocity fluctuations at $x=1.0$.

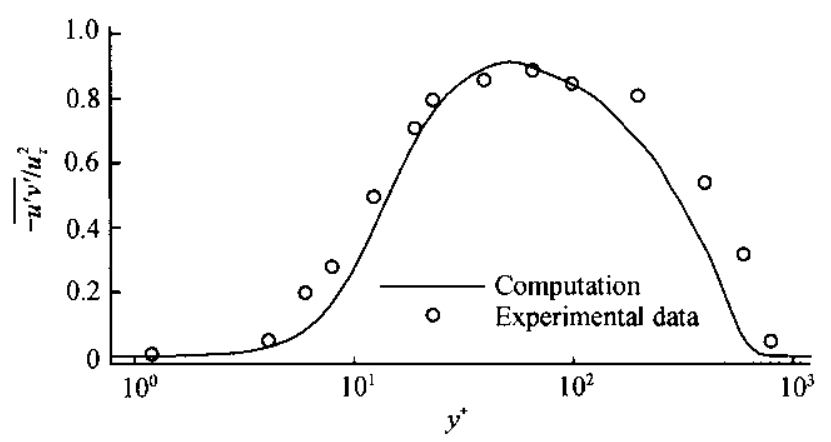

Fig. 15. Normalized Reynolds shear stress.

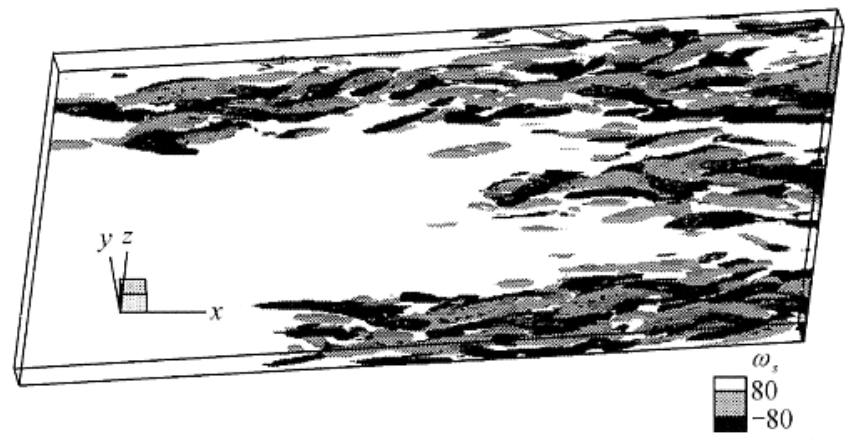

Fig. 16. Isosurface of streamwise vorticity for $\omega_{s}= \pm 80$ in the range $0.03 \leqslant x \leqslant 0.23$.

blunt-wedge $(x<-0.1)$, and the transition is finished at the body of the blunt-wedge. This figure shows that the near-wall disturbance is strongly suppressed at the nose of the blunt wedge, but the suppression of disturbance at the nose of the blunt wedge far from the wall is not significant.

Spanwise vorticity contours on the plane $y=0.0002,0.00043,0.00102$ and 0.00180 


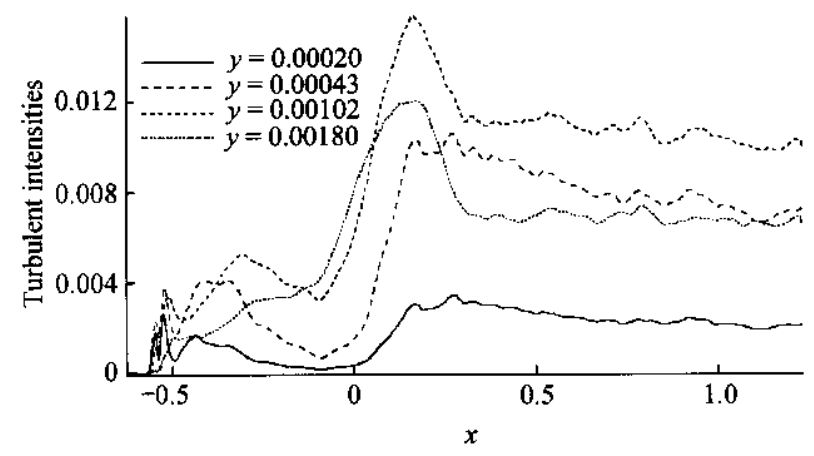

Fig. 17. Turbulent intensity as a function of $x$.

are shown in figs. $18-21$, in which the near-wall streaky structures can be seen clearly. Fig. 18 shows that at $x=-0.1$, the near-wall streaky structures are depressed, and this depression is not strong in the zone far from wall.

Streamwise vorticity contours on the plane $y=0.0002,0.00043,0.00102$ and 0.00180 are shown in figs. $22-25$, from which we can see that streamwise vorticity is depressed at $x=-0.1$, and the depression is strong in the near-wall zone.

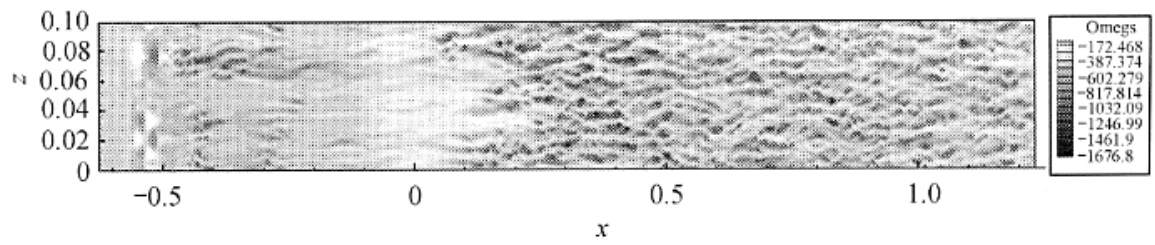

Fig. 18. Contour of spanwise vorticity at $y=0.0002$.

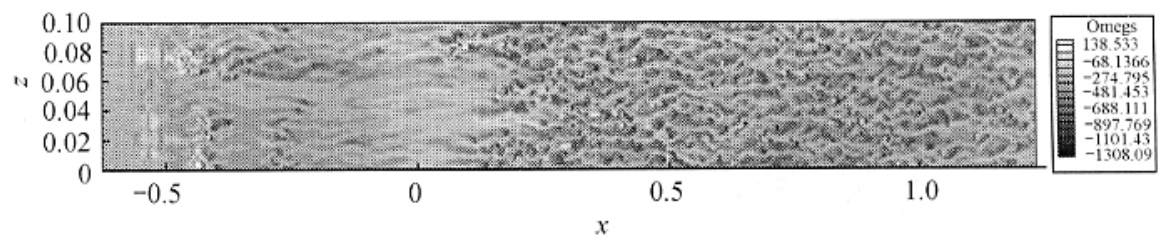

Fig. 19. Contour of spanwise vorticity at $y=0.00043$.

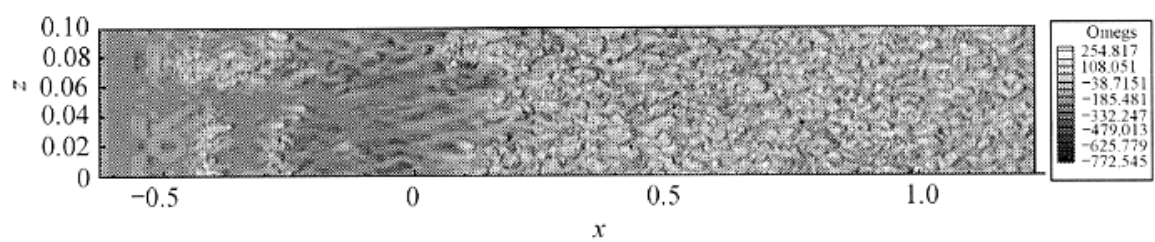

Fig. 20. Contour of spanwise vorticity at $y=0.00102$. 


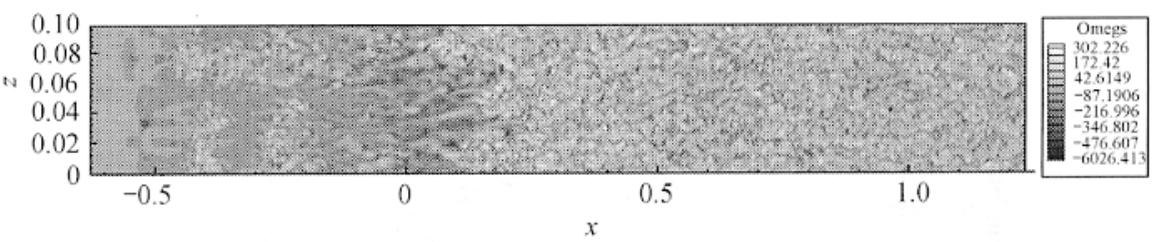

Fig. 21. Contour of spanwise vorticity at $y=0.00180$.

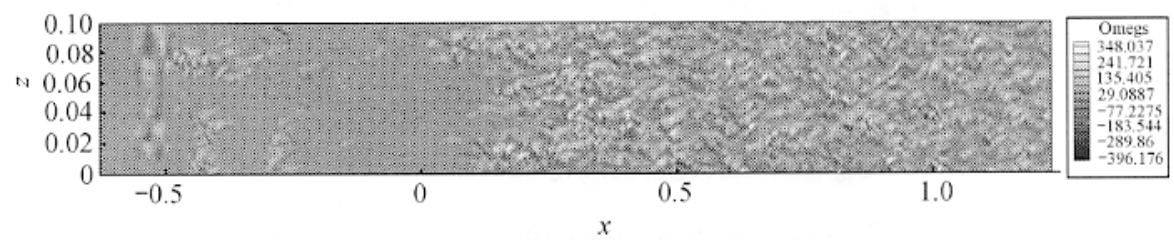

Fig. 22. Contour of streamwise vorticity at $y=0.0002$.

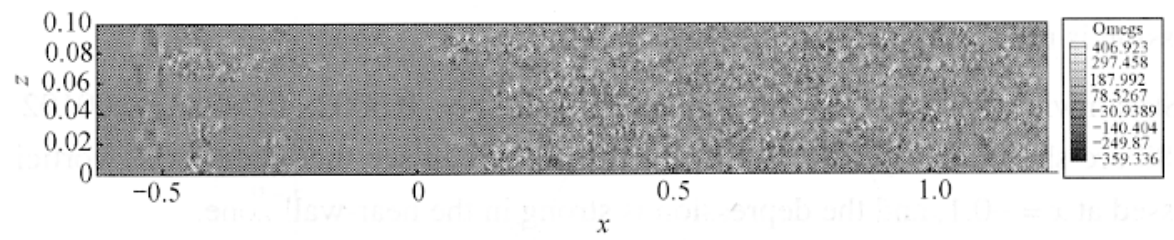

Fig. 23. Contour of streamwise vorticity at $y=0.00043$.

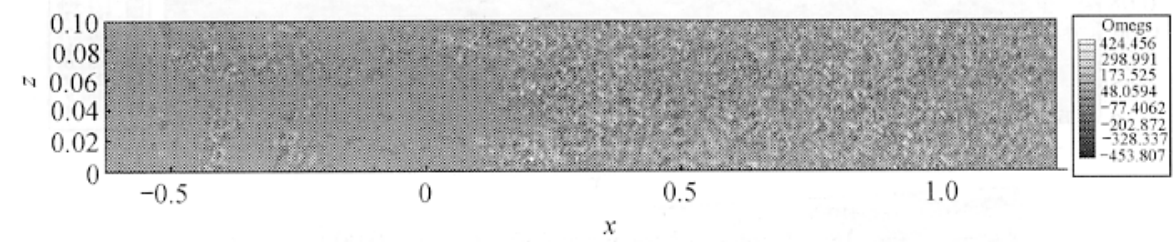

Fig. 24. Contour of streamwise vorticity at $y=0.00102$.

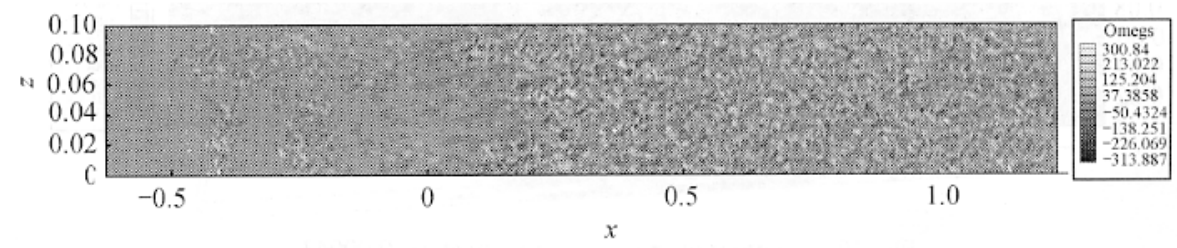

Fig. 25. Contour of streamwise vorticity at $y=0.00180$.

\section{Conclusion}

In this paper, DNS of turbulent boundary layer of supersonic flow of $M_{\infty}=6$ over a blunt wedge is performed, and the following conclusions are drawn.

1) Because of the bow shock out of the boundary layer, the turbulent Mach number 
in the turbulent boundary layer is not very high, and the turbulent Mach number is much less than that in the flat plate boundary layer.

2) Because the turbulent Mach number is not high, the compressible effects are not significant.

3) In this flow, transition begins at the head of blunt wedge, and then the transition is depressed. Transition is finished at the body of the wedge. The near-wall disturbance is depressed strongly.

\section{Discussion}

From figs. 10, 17 and $18-25$, we can see that the transition is very fast, so it may be a bypass transition. We will study this transition later. It is also found that the base frequency has strong effect on transition, and high frequency disturbance will not generate transition.

Acknowledgements The authors would like to thank the State Key Laboratory of Scientific and Engineering Computing (LSEC) for providing computer time. The authors thank Professor Zhang Linbo of LSEC for the help in programming. This work was supported by NKBRSF (Grant No. CG199032805), and the National Natural Science Foundation of China (Grant Nos. 90205025, 19872069, 170176033), and the Informatization Construction of Knowledge Innovation Projects of the Chinese Academy of Sciences (Grant No. INF105-SCE)..

\section{References}

1. Guarini, S. E., Moser, R. D., Shariff, K. et al., Direct numerical simulation of a supersonic turbulent boundary layer at Mach 2.5, J. Fluid Mech., 2000, 414: 1-33.

2. Maeder, T., Adams, N. A., Kleiser, L., Direct simulation of turbulent supersonic boundary layers by an extended temporal approach, J. Fluid Mech., 2001, 429: 187-216.

3. Rai, M. M., Gatski, T. B., Erlebacher, G., Direct simulation of spatially evolving compressible turbulent boundary layers, AIAA Paper, 1995, 95-0583.

4. Zhong, X. L., Leading-edge receptivity to free-stream disturbance waves for hypersonic flow over a parabola, J. Fluid Mech., 2001, 441: 315-367.

5. Laurien, E., Numerical investigation of laminar-turbulent transition of re-entry capsules, Journal of Spacecraft and Rockets, 1996, 33(3): 313-318.

6. Liu, X. D., Osher, S., Chan, T., Weighted essentially non-oscillatory schemes, Journal of Computational Physics, 1994, 115: 200-212.

7. Liubimov, A. N., Rusanov, B. B., Flow field of gas over a blunt body, Science, Moscow (in Russian), 1970, $134-135$.

8. Li, X. L., Fu, D. X., Ma, Y. W., Direct numerical simulation of compressible isotropic turbulence, Science in China, Ser. A, 2002, 45(11): 1452-1460.

9. Fu, D. X., Computational Aerodynamics (in Chinese), Chapter 2, Beijing: Astronavigation Publishing Company, 1994.

10. Li, X. L., Ma, Y. W., Fu, D. X., DNS and scaling law analysis of compressible turbulent channel flow, Science in China, Ser. A, 2001, 44(5): 645-654. 